The Chinese Society of Theoretical and Applied Mechanics

Chinese Journal of Mechanics Press, Beijing, China

Allerton Press, INC., New York, U.S.A.

\title{
SIDEWALL FLOW STABILITY IN THE MHD CHANNEL FLOWS*
}

\author{
$\mathrm{Xu} \mathrm{Fu}$ (徐 复) \\ (Institute of Mechanics, Chinese Academy of Sciences, Beijing 100080, China)
}

\begin{abstract}
ABSTRAC'T: The velocity distribution between two sidewalls is M-shaped for the MHD channel flows with rectangular cross section and thin conducting walls in a strong transverse magnetic field. Assume that the dimensionless numbers $R_{m} \ll 1, M, N \gg 1$, and $\sigma^{*} \ll 1$ and that the distance between two perpendicular walls is very long in comparison with the distance between two sidewalls. First, the equation for steady flow is established, and the solution of M-shaped velocity distribution is given. Then, an equation for stability of small disturbances is derived based on the velocity distribution obtained. Finally, it is proved that the stability equation for sidewall flow can be transformed into the famous Orr-Sommerfeld equation, in addition, the following theorems are also proved, namely, the analogy theorem, the generalized Rayleigh's theorem, the generalized Fjortoft's theorem and the generalized Joseph's theorems.
\end{abstract}

KEY WORDS: MHD channel flow, sidewall flow, flow stability

The problem of sidewall flow stability of MHD channel flows with rectangular cross section and thin conducting walls under a strong magnetic field is discussed in this paper. The Cartesian coordinates oxyz are adopted. The $x$ direction is the flow direction, and the $y$ direction is the direction of magnetic field. The walls at $y= \pm a$ are called the perpendicular walls, while the walls at $z= \pm b$ are called the sidewalls or parallel walls. The effect of viscosity is mainly limited within the boundary layers. The thickness of perpendicular-wall boundary-layer is of the order of $a / M$, while the thickness of sidewall boundary-layer or side layer is of the order of $b / \sqrt{M}$, where $M$ is the Hartmann number ${ }^{[1 \sim 3]}$. The results obtained from both theory and experiments show that the velocity distribution $u(y=$ const, $z$ ) is M-shaped, namely, the wall jets appear in the side layers, and under certain conditions the flow rate within the two side layers may even take a certain portion of the total flow rate, for example, one fourth. The instability of sidewall flow has already been observed in experiments, and the critical Reynolds number $R_{c r}$ has also been measured. ${ }^{[3]}$

Assume $a / b \gg 1$, and the problem of sidewall flow stability is solved approximately in the $o x z$ plane. In fluid mechanics, the critical Reynolds number $R_{\mathrm{cr}}$ is comparatively small

Received 24 August 1995, revised 2 February 1996

* The project supported by the National Natural Science Foundation of China 
for the velocity profile with inflection point, such as in some jets. However, in the sidewall flow, on the one hand the flow velocity profile has inflection points, and on the other hand the strong magnetic field has strong stabilizing effect on the flow, therefore, its critical Reynolds number may be comparatively large. The experimental data give ${ }^{[3]}: 2650<R_{\mathrm{cr}}<5100$ for $2700<M<5400$.

\section{MATHEMATICAL FORMULATION}

Divide the space into three regions: the one occupied by the conducting fluid inside the channel, the one occupied by the channel walls and the one occupied by the rest of dielectric medium outside the walls. In the region of conducting fluid, the governing equations are the MHD equations for the incompressible, viscous and conducting fluid flow in an uniform, applied magnetic field. In the region of channel walls, the governing equations are the equations of electrodynamics for conducting medium without the displacement current. Similarly, in the region of the rest of dielectric medium the equations of static magnetic field are the governing ones. Besides, at the two interfaces among the three regions, there are some boundary conditions ${ }^{[1,2,4]}$. The problem has already been solved theoretically ${ }^{[1,2]}$. Assume that:

(1) All parameters of physical properties are constants, for example $\rho, \eta, \nu(=\eta / \rho), \mu$, $\epsilon, \sigma$ of conducting fluid, etc.;

(2) The uniform, strong, transverse magnetic field is $\boldsymbol{B}_{0}=\left(0, B_{y o}, 0\right)$;

(3) The magnetic Reynolds number of the conducting fluid $R_{m}=\mu \sigma U_{0} b \ll 1$;

(4) The Hartmann number $M=B_{y 0} b \cdot \sqrt{\frac{\sigma}{\eta}} \gg 1$, and the interaction parameter $N=\frac{\sigma B_{y 0}^{2} b}{\rho U_{0}} \gg 1$

(5) The conductance ratio $\sigma^{*}=\frac{\sigma_{w} t_{w}}{\sigma b} \ll 1$ for thin conducting walls, where $\sigma_{w}, t_{w}$ are the electric conductivity and thickness of conducting walls respectively. Moreover, suppose that

$$
\sigma^{*}=O\left(M^{-1 / 2}\right) \quad \text { or } \quad M^{-1 / 2} \ll \sigma^{*} \ll 1
$$

Firstly, the equation of steady flow will be established and its solution will be given. Under the assumption of $R_{m} \ll 1$, the induced magnetic field can be neglected. The equation of $x$ direction momentum is

$$
-\frac{\partial \bar{p}}{\partial x}+\eta \frac{\mathrm{d}^{2} \bar{u}}{\mathrm{~d} z^{2}}-\sigma B_{y o}\left[\bar{E}(z)+B_{y o} \bar{u}\right]=0
$$

with the boundary conditions

$$
\bar{u}=0 \quad \text { at } \quad z= \pm b
$$

The supplementary assumption is

(6) $\frac{\partial \bar{p}}{\partial x}$ is a constant and the electric field $\bar{E}(z)$ is known.

As mentioned above, the problem of channel flow with rectangular cross section and thin conducting walls in an uniform, strong, transverse magnetic field has already been 
solved, for example, in Ref.[1,2]. By the words "the electric field $\bar{E}(z)$ is known", we mean that $\bar{E}(z)$ is given as an approximation to the solution $\bar{E}(y, z)$ obtained in Ref.[1,2]. Substituting $\bar{E}(z)$ into the above equation, the unknown function $\bar{u}(z)$ can then be solved, and based on the solved basic flow $\bar{u}(z)$, the stability equation of small disturbances can then be established. Here, the velocity distribution $\bar{u}(\dot{z})$ is also an approximation to the solution $\bar{u}(y, z)$ given in Ref.[1,2]. Besides, the velocity distribution $\bar{u}(z)$ is M-shaped. Let us introduce the following dimensionless quantities

$$
\begin{array}{lll}
u=\frac{\bar{u}}{U_{0}} & \zeta=\frac{z}{b} & t=\frac{\bar{t} U_{0}}{b} \\
E=\bar{E} /\left(U_{0} B_{y o}\right) & P=-\frac{1}{\rho} \frac{\partial \bar{p}}{\partial x} \frac{b}{U_{0}^{2}} & R=\frac{U_{0} b}{\nu}
\end{array}
$$

where $U_{0}$ is the characteristic magnitude of velocity. Then, the basic equation and the boundary conditions are

$$
\begin{array}{llc}
\frac{\mathrm{d}^{2} u}{\mathrm{~d} \zeta^{2}}-M^{2} u+P R & -M^{2} E(\zeta)=0 \\
u=0 & \text { at } & \zeta= \pm 1
\end{array}
$$

The solution is

$$
u(\zeta)=\frac{P}{N}\left[1-\frac{\operatorname{ch} M \zeta}{\operatorname{ch} M}\right]+u^{*}(1)\left[\frac{u^{*}(\zeta)}{u^{*}(1)}-\frac{\operatorname{ch} M \zeta}{\operatorname{ch} M}\right]
$$

where the symmetric function $u^{*}(\zeta)$ is

$$
u^{*}(\zeta)=\operatorname{sh} M \zeta \cdot \int_{0}^{M \zeta} E\left(\frac{\xi}{M}\right) \operatorname{ch} \xi \mathrm{d} \xi-\operatorname{ch} M \zeta \cdot \int_{0}^{M \zeta} E\left(\frac{\xi}{M}\right) \operatorname{sh} \xi \mathrm{d} \xi
$$

Now, let us derive the stability equation of small disturbances. Suppose

$$
\boldsymbol{u}=(U(\zeta)+\tilde{u}(\xi, \zeta, t), 0, \tilde{w}(\xi, \zeta, t))
$$

where $\xi=x / b, U(\zeta)$ is the M-shaped velocity distribution mentioned above, and $\tilde{u}, \tilde{w}$ are the disturbed velocities in the $x$ and $z$ direction respectively. Introducing $\phi(\xi, \zeta, t)$ and $\psi(\zeta)$, and supposing that

$$
\begin{aligned}
& \tilde{u}=\frac{\partial \phi}{\partial \zeta} \quad \tilde{w}=-\frac{\partial \phi}{\partial \xi} \\
& \phi(\xi, \zeta, t)=\psi(\zeta) \exp [\mathrm{i} \alpha(\xi-c t)]
\end{aligned}
$$

then the linearlized disturbed equation for $\psi(\zeta)$ is

$$
L_{1} \psi+\left(c+\mathrm{i} \frac{N}{\alpha}\right) L_{2} \psi=0
$$

where

$$
\begin{aligned}
& L_{1}=\frac{1}{\mathrm{i} \alpha R}\left(D^{2}-\alpha^{2}\right)^{2}-U(\zeta)\left(D^{2}-\alpha^{2}\right)+D^{2} U(\zeta) \\
& L_{2}=D^{2}-\alpha^{2} \quad \text { and } \quad D=\frac{\mathrm{d}}{\mathrm{d} \zeta}
\end{aligned}
$$


The boundary conditions are

$$
\psi=D \psi=0 \quad \text { at } \quad \zeta= \pm 1
$$

Note that, as $N=0$, the equation reduces to the well-known Orr-Sommerfeld equation ${ }^{[5]}$.

\section{SEVERAL THEOREMS ON THE SIDEWALL FLOW STABILITY}

Several theorems will be proved in the following in order to discuss the problem of sidewall flow stability qualitatively.

Suppose that the fluid flow is subjected to certain fictitious, unchanged, applied force field, and its velocity distribution is $U(\zeta)$ of M-shape. Then, the problem of flow stability is reduced to the eigenvalue problem of the Orr-Sommerfeld equation

$$
\begin{array}{ll}
L_{1} \bar{\psi}+\bar{c} L_{2} \bar{\psi}=0 & \text { at } \quad \zeta= \pm 1 \\
\bar{\psi}=D \bar{\psi}=0 \quad
\end{array}
$$

Analogy theorem For the problem (M) of sidewall flow stability with velocity distribution $U(\zeta)$, there corresponds to a problem $(F)$ of parallel flow stability in fluid mechanics with the same velocity distribution $U(\zeta)$. Once the problem $(\mathrm{F})$ is solved, and the eigenvalues and eigenfunctions are found to be

$$
\bar{c}=f(\alpha, R)
$$

then the eigenvalues and eigenfunctions for the problem (M) are

$$
c=f(\alpha, R)-\mathrm{i} \frac{N}{\alpha} \quad \psi(\zeta)=\bar{\psi}(\zeta)
$$

Separating the real part from the imaginary part of eigenvalues, the relations of eigenvalues between the two flows are then obtained

$$
\begin{aligned}
& c_{r}=\bar{c}_{r} \\
& c_{i}=\bar{c}_{i}-\frac{N}{\alpha} \quad \text { or } \quad \omega=\bar{\omega}-N
\end{aligned}
$$

where $\omega=\alpha c_{i}, \bar{\omega}=\alpha \bar{c}_{i}$ are the instability growth rates.

By this theorem, the stability equation for sidewall flow can be transformed into the Orr-Sommerfeld equation.

Now, let us discuss the case of inviscid flow $(R \rightarrow \infty)$ of sidewall flow stability. The problem (M) is simplified to the problem (MI):

$$
\begin{aligned}
& {\left[U(\zeta)-\left(c+\mathrm{i} \frac{N}{\alpha}\right)\right]\left[D^{2} \psi-\alpha^{2} \psi\right]-U^{\prime \prime}(\zeta) \psi=0} \\
& \psi=0 \quad \text { at } \quad \zeta= \pm 1
\end{aligned}
$$

Let $\bar{c}=c+\mathrm{i} N / \alpha$, then the equation is the Rayleigh equation ${ }^{[5]}$. 
Generalized Rayleigh's theorem One necessary condition for the instability of inviscid sidewall flow is that the velocity profile must have an inflection point $\zeta_{0}$, i.e., $U^{\prime \prime}\left(\zeta_{0}\right)=0,-1<\zeta_{0}<1$.

Proof Let $c=c_{r}+\mathrm{i} c_{i}$, and suppose $c_{i}>0$, then the equation of problem (MI) has no singularity. Multiplying the equation by the function $\psi^{*}(\zeta)$, the conjugate function of $\psi(\zeta)$, and integrating $\zeta$ from -1 to +1 , we have

$$
\int_{-1}^{+1}\left(\left|\psi^{\prime}\right|^{2}+\alpha^{2}|\psi|^{2}\right) \mathrm{d} \zeta+\int_{-1}^{+1} \frac{U^{\prime \prime}(\zeta)}{U-(c+\mathrm{i} N / \alpha)}|\psi|^{2} \mathrm{~d} \zeta=0
$$

The imaginary part of the equality is

$$
\left(c_{i}+\frac{N}{\alpha}\right) \cdot \int_{-1}^{+1} \frac{U^{\prime \prime}(\zeta)}{|U-(c+\mathrm{i} N / \alpha)|^{2}}|\psi|^{2} \mathrm{~d} \zeta=0
$$

From this equality, it is inferred that $U^{\prime \prime}(\zeta)$ must change its sign at least once in the interval $(-1,+1)$, i.e., there is at least one inflection point $\zeta_{0}$, satisfying $U^{\prime \prime}\left(\zeta_{0}\right)=0,-1<\zeta_{0}<+1$.

Since the velocity profile of $M$-shape includes at least two inflection points, the sidewall flow already satisfies the necessary condition for instability by this theorem.

Generalized Fj申rtoft's theorem One necessary condition for the instability of inviscid sidewall flow is that at certain point $\zeta$ within the flow field, the velocity profile satisfies the inequality $U^{\prime \prime}(\zeta)\left[U(\zeta)-U_{0}\right]<0$, where $U_{0}=U\left(\zeta_{0}\right)$ and $\zeta_{0}$ satisfies $U^{\prime \prime}\left(\zeta_{0}\right)=0$, $-1<\zeta_{0}<+1$.

Proof The real part of the integral equality in the above theorem is

$$
\int_{-1}^{+1} \frac{U^{\prime \prime} \cdot\left(U-c_{r}\right)}{|U-(c+\mathrm{i} N / \alpha)|^{2}}|\psi|^{2} \mathrm{~d} \zeta=-\int_{-1}^{+1}\left(\left|\psi^{\prime}\right|^{2}+\alpha^{2}|\psi|^{2}\right) \mathrm{d} \zeta
$$

Adding the following equality to the above equation,

$$
\left(c_{r}-U_{0}\right) \cdot \int_{-1}^{+1} \frac{U^{\prime \prime}(\zeta)}{|U-(c+\mathrm{i} N / \alpha)|^{2}}|\psi|^{2} \mathrm{~d} \zeta=0
$$

we have

$$
\int_{-1}^{+1} \frac{U^{\prime \prime}(\zeta)\left[U(\zeta)-U_{0}\right]}{|U-(c+\mathrm{i} N / \alpha)|^{2}}|\psi|^{2} \mathrm{~d} \zeta=-\int_{-1}^{+1}\left(\left|\psi^{\prime}\right|^{2}+\alpha^{2}|\psi|^{2}\right) \mathrm{d} \zeta<0
$$

Therefore, at certain point $\zeta$ within the flow field, we have

$$
U^{\prime \prime}(\zeta) \cdot\left[U(\zeta)-U_{0}\right]<0
$$

The proof is then completed. If $U(\zeta)$ has only one inflection point $\zeta_{0}$, and $U(\zeta)$ is a monotonic function, then one necessary condition for instability is $U^{\prime \prime}(\zeta)\left[U(\zeta)-U_{0}\right] \leq 0$ for certain $\zeta,-1 \leq \zeta \leq+1$, and the sign of equality holds only at the point $\zeta=\zeta_{0}$.

Now, let us return to the problem of viscous flow. The Joseph's theorems will then be extended to the case of sidewall flow stability, and the estimation on the eigenvalues $c_{i}$ and $c_{r}$ will be given. Notice that, the Joseph's theorems are only proved for the case of small 
Reynolds number $R$. Therefore, we suppose beforehand that the Reynolds number $R$ and the interaction parameter $N$ are small in the extension.

Joseph's first theorem ${ }^{[5 \sim 7]}$ Suppose $\bar{c}(\alpha, R)$ is an eigenvalue for the eigenvalue problem of the Orr-Sommerfeld equation (F), then the following inequality holds

$$
\bar{c}_{i} \leq \frac{q}{2 \alpha}-\frac{1}{\alpha R}\left[\frac{\pi^{2}\left(\pi^{2}+\alpha^{2}\right)}{\pi^{2}+4 \alpha^{2}}+\alpha^{2}\right]
$$

and there is no amplified disturbance $\left(\bar{c}_{i}>0\right)$ if the following inequality holds

$$
\alpha R q<f(\alpha)=\max \left[f_{1}(\alpha), f_{2}(\alpha)\right]
$$

where

$$
\begin{array}{ll}
q=\max \left|U^{\prime}(\zeta)\right| & -1 \leq \zeta \leq+1 \\
f_{1}(\alpha)=\lambda_{3}^{2} \pi^{2}+2^{3 / 2} \cdot \alpha^{3} & f_{2}(\alpha)=\left(\lambda_{3}^{2}+\alpha^{2}\right) \cdot \pi
\end{array}
$$

Now, let us extend this theorem to the case of sidewall flow stability. We have

Generalized Joseph's first theorem Suppose $c$ is an eigenvalue for the problem (M) of sidewall flow stability, then

$$
c_{i} \leq \frac{q}{2 \alpha}-\frac{1}{\alpha R}\left[\frac{\pi^{2}\left(\pi^{2}+\alpha^{2}\right)}{\pi^{2}+4 \alpha^{2}}+\alpha^{2}+M^{2}\right]
$$

and there is no amplified disturbance $\left(c_{i}>0\right)$ if the following inequality holds

$$
R<\frac{2}{q}\left[\frac{\pi^{2}\left(\pi^{2}+\alpha\right)}{\pi^{2}+4 \alpha^{2}}+\alpha^{2}+M^{2}\right]
$$

Proof From the analogy theorem, we have $\bar{c}_{i}=c_{i}+\frac{N}{\alpha}=c_{i}+\frac{M^{2}}{\alpha R}$. Inserting it to the Joseph's first theorem, then the theorem is proved.

Joseph's second theorem ${ }^{[5 \sim 8]}$ Suppose $\bar{c}(\alpha, R)$ is an eigenvalue of the eigenvalue problem (F) for the Orr-Sommerfeld equation

(1) If $U_{\min }^{\prime \prime} \geq 0$, then

$$
U_{\min }<\bar{c}_{r}<U_{\max }+\frac{2 U_{\max }^{\prime \prime}}{\pi^{2}+4 \alpha^{2}}
$$

(2) If $U_{\min }^{\prime \prime} \leq 0 \leq U_{\max }^{\prime \prime}$, then

$$
U_{\min }+\frac{2 U_{\min }^{\prime \prime}}{\pi^{2}+4 \alpha^{2}}<\bar{c}_{r}<U_{\max }+\frac{2 U_{\max }^{\prime \prime}}{\pi^{2}+4 \alpha^{2}}
$$

(3) If $U_{\max }^{\prime \prime} \leq 0$, then

$$
U_{\min }+\frac{2 U_{\min }^{\prime \prime}}{\pi^{2}+4 \alpha^{2}}<\bar{c}_{r}<U_{\max }
$$

where the subscripts $\min$, max indicate the minimum and maximum of the functions $U(\zeta), U^{\prime \prime}(\zeta)$ in the interval $-1 \leq \zeta \leq+1$ respectively. 
From the analogy theorem, we have $c_{r}=\bar{c}_{r}$, therefore the theorem can be applied to the case of sidewall flow stability directly.

If the MHD sidewall flow and the parallel flow in fluid mechanics have the same velocity profile $U(\zeta)$ which is symmetric and has inflection points, besides, if both $R$ and $N$ are small, then owing to the stabilizing effect of magnetic field on the sidewall flow, a conclusion can be draw from the generalized Joseph's first theorem, namely, the Reynolds number $R$ at which the sidewall flow still keeps stable is greater than the Reynolds number $R$ at which the fluid flow still keeps stable, the value of the difference is $\frac{2}{q} M^{2}$.

\section{REFEIIINCES}

1 Walker JE. Magnetohydrodynamic flows in rectangular ducts with thin conducting walls, Part 1. J de Méchanique, 1981, 20(1): 79 112

2 Sidorenkov S, Shishko A. Variational method for MHD flow calculation in conducting walls slotted channel. Magnetohydrodynamics, 1991, (4): 87 96

3 Reed CB, Picologlou BF. Sidewall flow instabilities in liquid metal MHD flows under blanket relevant conditions. Fusion Technology, 1989, 15: 705 715

4 Moreau R. Magnetohydrodynamics. Dordrecht: Kluwer Academic Publishers, 1990

5 Drazin PG, Reid WH. Hydrodynamic Stability. London: Cambridge University Press, 1981

6 Joseph DD. Eigenvalue bounds for the Orr-Sommerfeld equation, Part 1. J Fluid Mech, 1968, 33(3): $617 \sim 621$

7 Joseph DD. Eigenvalue bounds for the Orr-Sommerfeld equation, Part 2. J Fluid Mech, 1969, 36(4): 721 734

8 Georgescu A. Hydrodynamic Stability Theory. Dordrecht: Martinus Nijhoff Publishers, 1985 